\title{
Controle associado de Alphitobius diaperinus e efeito de microrganismos eficazes no desenvolvimento de Beauveria bassiana
}

\author{
Patricia Helena Santoro(1), Pedro Manuel Oliveira Janeiro Neves ${ }^{(1)}$, Silvia Akimi Cavaguchi( ${ }^{(1)}$, Kelly Constanski $^{(1)}$, \\ Junio Tavares Amaro(1), Luis Francisco Angeli Alves ${ }^{(2)}$ e Bruno Biazotto Gomes ${ }^{(1)}$
}

(1)Universidade Estadual de Londrina, Centro de Ciências Agrárias, Caixa Postal 6001, CEP 86051-990 Londrina, PR. E-mail:
ph_santoro@yahoo.com.br, pedroneves@uel.br, silvia_akimi@yahoo.com.br, kconstanski@hotmail.com, juniotavares@ibestvip.com.br,
bruno_biazotto@hotmail.com (2)Universidade do Oeste do Paraná, Rua Universidade 2069, CEP 85814-110 Cascavel, PR. Ifaalves@unioeste.br

\begin{abstract}
Resumo - O objetivo deste trabalho foi avaliar a eficiência de Beauveria bassiana $(\mathrm{Bb})$, terra diatomácea (TD) e microrganismos eficazes (EM-4), associados ou não, no controle de Alphitobius diaperinus, e o efeito de EM-4 no desenvolvimento de $B$. bassiana. Os agentes de controle (Bb, TD e EM-4), em diferentes concentrações e combinações, foram aplicados em uma mistura de cama-de-frango e ração, em que os insetos foram acondicionados por dez dias. Avaliaram-se, in vitro: a germinação, as unidades formadoras de colônia (UFC), o crescimento vegetativo e a produtividade de conídios de Bb em contato com suspensão aquosa de EM-4 (1\%) não filtrada e filtrada. Os maiores índices de mortalidade foram observados nos tratamentos TD + Bb + EM-4 e TD + Bb, nas maiores concentrações. Verificou-se efeito não-aditivo sinérgico para TD + Bb nas três concentrações. Nos testes com EM-4 filtrado, não houve diferença em relação à testemunha quanto à germinação e às UFC, entretanto, $\mathrm{o}$ crescimento vegetativo e a produtividade de conídios foram negativamente afetados. O uso conjunto de B. bassiana e terra diatomácea, para o manejo de populações de A. diaperinus, pode reduzir o uso de produtos químicos.
\end{abstract}

Termos para indexação: avicultura, cascudinho, controle biológico, entomopatógeno, Tenebrionidae.

\section{Associated control of Alphitobius diaperinus and effect of effective microorganisms in the development of Beauveria bassiana}

\begin{abstract}
The objective of this work was to evaluate Beauveria bassiana (Bb), diatomaceous earth (TD) and effective microorganisms (EM-4), associated or not, to Alphitobius diaperinus control, and the EM-4 effect on B. bassiana development. The control agents (Bb, TD and EM-4), in different concentrations and combinations, were applied in a mixture of litter and chicken food, where insects had been left per ten days. Conidia germination, colony-forming units (CFU), vegetative growth and the productivity of $\mathrm{Bb}$ conidia were evaluated when in contact with a filtered and non filtered watery EM-4 suspension (1\%). The highest indices of mortality were observed in the treatments $\mathrm{TD}+\mathrm{Bb}+\mathrm{EM}$ and $\mathrm{TD}+\mathrm{Bb}$ in the highest concentrations. A synergic non additive effect was observed for TD $+\mathrm{Bb}$ in the three concentrations. In filtered EM-4 tests, no difference was observed related to the control of germination and CFU, however, vegetative growth and conidia productivity were negatively affected. The joint use of B. bassiana and diatomaceous earth for A. diaperinus populations management can minimize the use of chemical products.
\end{abstract}

Index terms: poultry keeping, darkling beetle, biological control, entomopathogen, Tenebrionidae.

\section{Introdução}

Os avanços na avicultura, obtidos pelo melhoramento genético dos animais, nutrição e manejo, resultaram em curtos períodos de criação, porém em condições que comprometem o bem-estar das aves e a eficiência dos sistemas de produção. Entre os problemas, pode-se citar a ocorrência, em grandes populações, de Alphitobius diaperinus (Panzer) (Coleoptera: Tenebrionidae), também conhecido como cascudinho. Esse inseto, anteriormente citado como praga de grãos armazenados, foi introduzido nos aviários junto à ração destinada à alimentação das aves e adaptou-se às condições dos sistemas de criação, onde se tornou a principal praga 
(O’Connor, 1987). Além de ser vetor de diversas doenças que atacam as aves e os homens, esse inseto serve de alimento alternativo, com isso, diminui o consumo de ração e conseqüentemente o ganho de peso dos animais (Axtell \& Arends, 1990).

A presença constante das aves nos aviários dificulta o controle desses insetos com agrotóxicos, que podem causar a intoxicação dos animais (Alves et al., 2005). A ocorrência natural de fungos entomopatogênicos, como Beauveria bassiana (Bals.), na praga, sugere o potencial de controle desses patógenos, principalmente em razão das condições de temperatura e umidade da cama-de-frango, que podem favorecer a ocorrência de epizootias dos fungos (Alves et al., 2005). Além disso, os fungos entomopatogênicos são inócuos aos animais endotérmicos (Alves, 1998), e podem ser utilizados nos aviários durante todo o desenvolvimento dos animais.

Outro produto de origem orgânica, que não produz resíduos tóxicos, é a terra diatomácea, utilizada principalmente no controle de pragas em grãos armazenados (Korunic, 1998; Lorini, 2001). Terra diatomácea é um sedimento amorfo que se origina de organismos unicelulares vegetais, como algas microscópicas aquáticas, marinhas e lacustres (Andrade et al., 2001), que provoca danos à cutícula dos insetos pela adsorção da cera da epicutícula e pela abrasão da cutícula, o que a torna permeável à água e promove a morte por dessecação (Korunic, 1998). A atividade prolongada dos pós inertes, aliada à incapacidade dos insetos de desenvolver resistência a eles, os tornam potencialmente competitivos para o controle de pragas, além disso, o uso de terra diatomácea no controle de insetos tende a se tornar muito comum em regiões de clima seco em razão da melhoria de sua eficiência (Atui et al., 2003).

O composto microbiano, originalmente denominado microrganismos eficazes (EM-4), vem sendo utilizado em aviários, com o objetivo de alterar a composição microbiana da cama-de-frango e diminuir a quantidade de amônia para torná-la inadequada ao desenvolvimento do cascudinho. Sua incorporação nos sistemas de criação de aves é feita pela pulverização sobre a cama-defrango, ou ainda, misturado à água dos bebedouros, para fornecer aos animais vitaminas e antibióticos. Esse produto é composto por bactérias ácido-láticas (Lactobacillus e Pediococcus), leveduras (Sacharomyces), bactérias fotossintéticas e actinomicetos, todos compatíveis entre si (Higa \& Parr, 1994). No Brasil, é produzido pela Fundação Mokiti
Okada e utilizado também nos cultivos de hortaliças, cereais, frutas e flores. Esses microrganismos produzem ácidos orgânicos, hormônios vegetais (giberelinas, auxinas e citocininas), além de vitaminas, antibióticos e polissacarídeos (Higa, 1992), no entanto, não são conhecidos relatos sobre sua eficiência para o controle de pragas.

Em sistemas onde se pretende utilizar métodos alternativos de controle de pragas, como a utilização de B. bassiana, terra diatomácea e EM-4, é importante conhecer o efeito conjunto destes agentes, principalmente a relação entre $B$. bassiana e EM-4, por se tratar de microrganismos que podem competir entre si.

O objetivo deste trabalho foi avaliar a eficiência de B. bassiana $(\mathrm{Bb})$, terra diatomácea (TD) e microrganismos eficazes (EM-4), associados ou não, no controle de A. diaperinus, e o efeito de EM-4 no desenvolvimento in vitro de $B$. bassiana.

\section{Material e Métodos}

O experimento foi realizado com 20 mil insetos adultos da espécie $A$. diaperinus coletados em aviário comercial no Município de Londrina, PR. Em laboratório, os insetos foram separados da cama-de-frango e acondicionados em bandejas com ração, até a instalação do experimento, por cerca de 24 horas. A cama-de-frango utilizada no experimento, como substrato para os insetos, foi coletada no mesmo aviário, era constituída de maravalha e foi reutilizada pelo segundo lote consecutivo de aves. Para a retirada dos insetos, fezes e outros materiais, e para a homogeneização do tamanho das partículas, a camade-frango foi peneirada (malha de $1 \mathrm{~mm}$ ). Em caixas

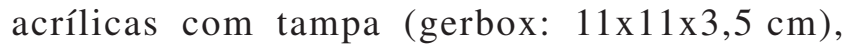
adicionaram-se $58 \mathrm{~g}$ de cama-de-frango peneirada e $2 \mathrm{~g}$ de ração para aves para alimentar os insetos durante o experimento.

Os agentes de controle de A. diaperinus, testados de maneira isolada ou associados dois a dois e três a três, foram: conídios do isolado Unioeste 4 de B. bassiana, previamente selecionado para o controle de $A$. diaperinus (Rohde et al., 2006); terra diatomácea comercial, composta por $86 \%$ de dióxido de sílica amorfa, com partículas de aproximadamente $15 \mu \mathrm{m}$; e EM-4.

As aplicações de terra diatomácea e B. bassiana foram realizadas apenas no primeiro dia, misturadas ao substrato na forma sólida (conídios do fungo e/ou partículas de terra diatomácea). As concentrações de 
terra diatomácea utilizadas foram: 0,5, 1,0 e 1,5\%, em relação ao peso total do substrato de cada gerbox $(60 \mathrm{~g})$. Essas concentrações foram definidas com base em prétestes, em que a maior concentração $(1,5 \%)$ foi responsável por um controle de aproximadamente $6 \%$ da população de insetos.Utilizaram-se conídios de B. bassiana produzidos em meio BDA, com viabilidade superior a $97 \%$ em teste de germinação; as concentrações no experimento foram: 0,02, 0,04 e 0,08\% do peso total do substrato. Essas concentrações também foram estabelecidas em pré-testes, em que a maior concentração $(0,08 \%)$ correspondeu à mortalidade de aproximadamente $20 \%$ dos indivíduos. Como um dos objetivos do trabalho foi avaliar a ação conjunta dos agentes de controle, optou-se por utilizar concentrações capazes de causar baixa mortalidade e, assim, evidenciar a interação, positiva ou negativa, entre os agentes de controle. Por meio de cálculos matemáticos, verificouse que, para o método de Koppenhofer et al. (2000), quando se utilizam concentrações em que a soma da percentagem de mortalidade dos agentes $\left(M_{1}+M_{2}\right)$ é igual ou superior a $116 \%$, não é possível visualizar os efeitos não-aditivos, pois os valores de $\chi^{2}$ calculado serão sempre inferiores ao tabelado, visto que a mortalidade máxima a ser obtida na associação dos agentes $\left(\mathrm{M}_{12}\right)$ não ultrapassa o valor de $100 \%$.

Para ser utilizado no experimento, o EM-4 foi ativado segundo a recomendação do fabricante, em que EM-4, melaço de cana-de-açúcar e água destilada esterilizada foram misturados nas proporções, em volume, de 1:1:8. A mistura foi mantida a $35^{\circ} \mathrm{C}$, e o produto da fermentação, decorrente do processo de ativação, foi diluído em água destilada esterilizada a $1 \%\left(\mathrm{~V} \mathrm{~V}^{-1}\right)$, tendo sido utilizada do terceiro ao nono dia após o início da ativação. A aplicação do EM-4 foi feita por pulverizações diárias sobre a cama-de-frango. A dose recomendada para a aplicação do EM-4 em aviários é feita com base na área do aviário; assim, para a conversão da dose em relação a peso de substrato, em cada gerbox (60 g), considerouse que $1 \mathrm{~m}^{2}$ a $0,20 \mathrm{~m}$ de profundidade de cama-defrango contém aproximadamente $15 \mathrm{~kg}$. As doses e as aplicações foram feitas em três etapas: no primeiro dia, realizou-se a pulverização de choque de $4 \mathrm{~mL}$ por gerbox sobre a cama-de-frango; no segundo dia, pulverizou-se 0,4 $\mathrm{mL}$; e do terceiro ao sétimo dia foram feitas pulverizações diárias de $0,16 \mathrm{~mL}$. Durante as pulverizações, a cama-de-frango foi revolvida para melhor incorporação do produto ao substrato. As aplicações foram feitas com pulverizador Airbrush, acoplado a um compressor-aspirador Fanen-Diapump na pressão de $0,8 \mathrm{kgf} \mathrm{cm}^{-1}$.

No tratamento testemunha não foi realizada a aplicação dos agentes de controle e os insetos foram mantidos no gerbox apenas com cama-de-frango e ração. Foram realizados 20 tratamentos, cada um com cinco repetições (gerbox) de 200 insetos. Os tratamentos foram mantidos em câmara climatizada $\left(25 \pm 1^{\circ} \mathrm{C}\right.$ e fotófase de 12 horas), e a avaliação foi realizada no décimo dia após o início da instalação do experimento, em que se quantificaram os insetos mortos.

Os dados de mortalidade total foram submetidos à análise de variância, e as médias comparadas pelo teste de Tukey, a 5\% de probabilidade. Antes de serem analisados estatisticamente pelo método proposto por Koppenhofer et al. (2000), os dados foram corrigidos pela fórmula de Shneider e Orelli (Nakano et al., 1981). O método de Koppenhofer et al. (2000) avalia a interação dos agentes de controle em associações, pelo teste de qui-quadrado, $\mathrm{e}$ considera $\chi^{2}=\left(M_{12}-M_{E}\right)^{2} / M_{E}$, em que $M_{12}$ é a mortalidade no tratamento onde foram aplicados os agentes de controle em associação; e $\mathrm{M}_{\mathrm{E}}$ é a mortalidade esperada, sendo $\mathrm{M}_{\mathrm{E}}=\mathrm{M}_{1}+\mathrm{M}_{2} \times\left(1-\mathrm{M}_{1} / 100\right)$, em que $\mathrm{M}_{1}$ e $\mathrm{M}_{2}$ representam a mortalidade nos tratamentos com apenas um dos agentes. Para o tratamento T18, em que foram associados três agentes de controle, considerou-se $\chi^{2}=\left(M_{123}-M_{E}\right)^{2} / M_{E}$ e $M_{E}=M_{1}+M_{2}+M_{3}$ $\left[\left(\mathrm{M}_{1} \times \mathrm{M}_{2} \times \mathrm{M}_{3}\right) / 100\right]$. Para $\chi^{2}$ significativo (3,84 para 1 grau de liberdade, $\mathrm{p}<0,05)$, o efeito foi considerado não-aditivo e sinérgico, se $\mathrm{M}_{12}-\mathrm{M}_{\mathrm{E}}<0$, ou antagônico se $M_{12}-M_{E}>0$. Se $\chi^{2}$ foi não-significativo, o efeito foi considerado aditivo.

As variáveis analisadas para estudar o efeito in vitro do EM-4, sobre conídios de B. bassiana, foram a germinação dos conídios, as unidades formadoras de colônias (UFC), o crescimento vegetativo e a produtividade de conídios. Para ser utilizado no experimento, o EM-4 foi ativado segundo o método descrito no experimento anterior, em que no terceiro dia após o início da ativação, foi diluído em água destilada esterilizada a $1 \%\left(\mathrm{~V} \mathrm{~V}^{-1}\right)$. Para avaliar a ação entre os microrganismos existentes no EM-4 e, também, os efeitos de seus metabólitos, os testes foram realizados com EM-4 sem filtrar e filtrado em membrana de $10 \mathrm{kd}$, pelo sistema de ultra-filtração Amicon, com pressão de nitrogênio de $100 \mathrm{kPa}$.

Para o teste de germinação utilizaram-se lâminas para microscópio $(2,5 \times 7,5 \mathrm{~cm})$ com $3 \mathrm{~mL}$ de meio BDA, em que foi pulverizado $0,1 \mathrm{~mL}$ da suspensão do fungo a 
$1 \times 10^{7}$ conídios $\mathrm{mL}^{-1}$, em solução aquosa de Tween $20 \mathrm{a}$ $0,02 \%\left(\mathrm{~V} \mathrm{~V}^{-1}\right)$, e $0,1 \mathrm{~mL}$ da calda de EM-4. A avaliação foi realizada 24 horas após a contaminação. Com auxílio de microscópio óptico, observaram-se aproximadamente 200 conídios, divididos em dois campos, e foram quantificados os germinados e os não germinados.

Para avaliação das unidades formadoras de colônias (UFC), pulverizou-se $0,1 \mathrm{~mL}$ de uma suspensão de conídios a $1 \times 10^{3}$ conídios $\mathrm{mL}^{-1}$, em solução aquosa de Tween 20 a $0,02 \%\left(\mathrm{~V} \mathrm{~V}^{-1}\right)$, e $0,1 \mathrm{~mL}$ de EM-4, sobre placas de Petri de $9 \mathrm{~cm}$ de diâmetro com $20 \mathrm{~mL}$ de meio BDA. A avaliação foi realizada oito dias após a contaminação, tendo-se quantificado as colônias formadas.

Nos testes de crescimento vegetativo e produtividade de conídios, pulverizou-se $0,1 \mathrm{~mL}$ de EM-4 sobre placas de Petri de $9 \mathrm{~cm}$ de diâmetro com aproximadamente $20 \mathrm{~mL}$ de meio BDA. Em seguida, o fungo foi colocado em três pontos eqüidistantes, com o auxílio de uma alça de platina. No 14o dia após a contaminação, calculou-se a área de cada colônia pela média obtida com as medidas dos diâmetros maior e menor, tendo-se escolhido uma colônia por placa, ao acaso. Dessas mesmas colônias, avaliouse a produtividade de conídios, recortando-se o centro delas com um vazador circular $\left(2,27 \mathrm{~cm}^{2}\right)$. Os discos de meio com o fungo foram suspensos em solução aquosa de Tween 20 a $0,02 \%\left(\mathrm{~V} \mathrm{~V}^{-1}\right)$ e submetidos à agitação por $30 \mathrm{~s}$ em vortex. Após as diluições necessárias, o número de conídios foi quantificado por meio de câmara de Neubauer.

As aplicações da suspensão de fungo e da calda de EM-4 ativado foram feitas com pulverizador Airbrush, acoplado a um compressor-aspirador Fanen-Diapump à pressão de $1 \mathrm{kgf} \mathrm{cm}^{-1}$. Todos os tratamentos foram mantidos em câmara climatizada $\left(25 \pm 1^{\circ} \mathrm{C}\right.$ e fotófase de 12 horas). $\mathrm{O}$ delineamento experimental foi inteiramente casualizado, com cinco repetições (placas) por tratamento. Para cada teste, foi feita uma testemunha em que se aplicou apenas solução aquosa de Tween 20 a $0,02 \%\left(\mathrm{~V} \mathrm{~V}^{-1}\right)$. Os dados foram submetidos à análise de variância, e as médias foram comparadas pelo teste de Tukey, a 5\% de probabilidade.

\section{Resultados e Discussão}

Verificou-se que no décimo dia da instalação do experimento, para a menor concentração dos agentes de controle, tanto em associação como isoladamente, os maiores índices de mortalidade de A. diaperinus foram observados nos tratamentos T7 (TD + Bb) com $25,20 \%$, seguido de T4 (Bb) com 13\% (Tabela 1). Para a concentração média, verificou-se maior controle em $\mathrm{T} 8(\mathrm{TD}+\mathrm{Bb})$ com $35,40 \%$, seguido de $\mathrm{T} 17 \mathrm{TD}+\mathrm{Bb}+$ EM-4) com 30,40\%. Na maior concentração, os melhores tratamentos foram $\mathrm{T} 18(\mathrm{TD}+\mathrm{Bb}+\mathrm{EM})$ e $\mathrm{T} 9$ $(\mathrm{TD}+\mathrm{Bb})$, com 49,80 e 41,40\%, respectivamente. Quanto à menor concentração e à concentração média, os tratamentos T10 e T11, que continham apenas TD + $\mathrm{EM}$, não diferiram da testemunha, com mortalidade de 1,4 e $4,6 \%$, respectivamente. Os tratamentos em que foi utilizada a terra diatomácea associada à $B$. bassiana foram sempre superiores, com níveis de mortalidade mais elevados.

Ao comparar a mortalidade nas diferentes concentrações, em relação aos agentes de controle, associados ou não, verificou-se que, nos tratamentos com $\mathrm{Bb}$ (T4, T5 e T6), TD + Bb (T7, T8 e T9), Bb + EM-4 (T13, T14 e T15) e TD + Bb + EM-4 (T16, T17 e T18), os índices de mortalidade foram diretamente proporcionais à concentração utilizada (Tabela 1). Para TD + EM-4 (T10, T11 e T12), os maiores índices de mortalidade foram observados nas duas maiores concentrações, que não diferiram estatisticamente entre si.

Nos tratamentos apenas com TD (T1, T2 e T3), não houve diferença no controle de A. diaperinus em relação às concentrações utilizadas, com mortalidade de 3,80 a $6,80 \%$; a menor concentração e a concentração média não diferiram da testemunha (Tabela 1). Esses resultados são inferiores aos de Alves et al. (2006), que ao utilizar quantidades inferiores de $\mathrm{TD}(0,1,0,2$ e $0,3 \%)$, misturadas à ração, observaram mortalidade de A. diaperinus entre 70 e $90 \%$ ao décimo dia de avaliação e à mesma temperatura. Esta diferença dos valores de mortalidade pode estar relacionada aos substratos utilizados nos diferentes estudos, ou então ao menor número de insetos utilizados por Alves et al. (2006).

A comparação entre os 20 tratamentos mostra que os maiores índices de mortalidade de A. diaperinus ocorreram nos tratamentos T18 (TD + Bb + EM-4: maior concentração), T9 (TD + Bb: maior concentração) e T8 (TD + Bb: concentração média), com 49,8, 41,4 e $35,4 \%$, respectivamente (Tabela 1 ). As menores taxas de mortalidade foram observadas nos tratamentos T1 (TD: menor concentração), T19 (EM-4), T10 (TD + EM-4: menor concentração), T11 (TD + EM-4: concentração média) e T12 (TD + EM: maior 
concentração), os quais não diferiram estatisticamente da testemunha, com mortalidade inferior a $5 \%$.

Quando se analisaram os valores médios de mortalidade corrigida, nas três concentrações, para cada agente de controle ou associação, verificou-se que o nível de controle em que se utilizou apenas $\mathrm{Bb}(16,73 \%)$ foi cinco vezes superior ao observado com TD $(3,27 \%)$ (Tabela 1). Na associação TD + Bb $(32,65 \%)$, a mortalidade foi dez vezes superior, em comparação à média com $\operatorname{TD}(3,27 \%)$, e aproximadamente duas vezes maior em relação à média com $\mathrm{Bb}(16,73 \%)$. Ao se comparar a associação TD + Bb (32,65\%) com TD + $\mathrm{Bb}+\mathrm{EM}-4$ (27,96\%), verificou-se redução na mortalidade de A. diaperinus, que mostrou efeito negativo de EM-4. O mesmo fato pode ser observado, quando o EM-4 foi associado à $B$. bassiana, e também à terra diatomácea.
O aumento da eficiência de controle de $A$. diaperinus promovido pela associação de $B$. bassiana com terra diatomácea reduz a quantidade necessária de cada agente, o que torna o controle economicamente mais viável. Incrementos da mortalidade, em associações de B. bassiana com terra diatomácea, também foram observados para pragas de grãos armazenados como Rhyzopertha dominica e Tribolium confusum (Vayas \& Athanassiou, 2004; Lord, 2005). Akbar et al. (2004) não verificaram diferença na mortalidade de Tribolium castaneum entre os tratamentos que contiveram apenas o fungo e em associação.

Ao se analisar a interação dos agentes de controle (Koppenhofer et al., 2000), verificou-se efeito nãoaditivo sinérgico nos tratamentos para as três concentrações de TD + Bb (T7, T8 e T10) (Tabela 1). Esses dados mostram que existe ganho

Tabela 1. Mortalidade total e corrigida de adultos de Alphitobius diaperinus submetidos à ação de diferentes agentes de controle biológico, associados ou não, e interação dos agentes em associação no décimo dia de avaliação ${ }^{(1)}$.

\begin{tabular}{|c|c|c|c|c|c|c|}
\hline \multirow[t]{2}{*}{ Tramento } & \multicolumn{3}{|c|}{ Agentes de controle ${ }^{(2)}$} & \multicolumn{2}{|c|}{ Mortalidade (\%) } & \multirow{2}{*}{$\begin{array}{c}\text { Interação dos agentes } \\
\text { de controle }\end{array}$} \\
\hline & TD & $\mathrm{Bb}$ & EM-4 & Total & Corrigida & \\
\hline $\mathrm{T} 1$ & 0,5 & - & - & $3,80 \pm 0,37 \mathrm{IJLa} D E$ & 1,84 & - \\
\hline $\mathrm{T} 2$ & 1,0 & - & - & $5,00 \pm 0,94 \mathrm{IJLa} E$ & 3,06 & - \\
\hline $\mathrm{T} 3$ & 1,5 & - & - & $6,80 \pm 0,92 \mathrm{IJa} E$ & 4,90 & - \\
\hline Média & - & - & - & 5,20 & 3,27 & - \\
\hline $\mathrm{T} 4$ & - & 0,02 & - & $13,00 \pm 0,95 \mathrm{Gc} B$ & 11,22 & - \\
\hline T5 & - & 0,04 & - & $18,80 \pm 1,46 \mathrm{Fb} C$ & 17,14 & - \\
\hline T6 & - & 0,08 & - & $23,40 \pm 1,12 \mathrm{EFaC}$ & 21,84 & - \\
\hline Média & - & - & - & 18,40 & 16,73 & - \\
\hline $\mathrm{T} 7$ & 0,5 & 0,02 & - & $25,20 \pm 1,16 \mathrm{Ec} A$ & 23,67 & Não-aditivo sinérgico \\
\hline $\mathrm{T} 8$ & 1,0 & 0,04 & - & $35,40 \pm 1,21 \mathrm{Cb} A$ & 34,08 & Não-aditivo sinérgico \\
\hline T9 & 1,5 & 0,08 & - & $41,40 \pm 0,98 \mathrm{Ba} B$ & 40,20 & Não-aditivo sinérgico \\
\hline Média & - & - & - & 34,00 & 32,65 & - \\
\hline $\mathrm{T} 10$ & 0,5 & - & EM-4 & $1,40 \pm 0,67 \mathrm{Lb} E$ & $-0,61$ & Aditivo \\
\hline $\mathrm{T} 11$ & 1,0 & - & EM-4 & $4,60 \pm 0,51 \mathrm{IJLa} E$ & 2,65 & Aditivo \\
\hline $\mathrm{T} 12$ & 1,5 & - & EM-4 & $5,00 \pm 0,55 \mathrm{IJLaEF}$ & 3,06 & Aditivo \\
\hline Média & - & - & - & 3,67 & 1,70 & - \\
\hline T13 & - & 0,02 & EM-4 & $7,40 \pm 0,68 \mathrm{HIc} C D$ & 5,51 & Aditivo \\
\hline $\mathrm{T} 14$ & - & 0,04 & EM-4 & $12,00 \pm 1,05 \mathrm{GHb} D$ & 10,20 & Aditivo \\
\hline $\mathrm{T} 15$ & - & 0,08 & EM-4 & $18,60 \pm 1,08 \mathrm{Fa} D$ & 16,94 & Aditivo \\
\hline Média & - & - & - & 12,67 & 10,88 & - \\
\hline T16 & 0,5 & 0,02 & EM-4 & $8,00 \pm 1,00 \mathrm{HIcC}$ & 6,12 & Aditivo \\
\hline $\mathrm{T} 17$ & 1,0 & 0,04 & EM-4 & $30,40 \pm 1,03 \mathrm{Db} B$ & 28,98 & Aditivo \\
\hline $\mathrm{T} 18$ & 1,5 & 0,08 & EM-4 & $49,80 \pm 1,39 \mathrm{Aa} A$ & 48,78 & Aditivo \\
\hline Média & - & - & - & 29,40 & 27,96 & - \\
\hline T19 & - & - & EM-4 & $1,60 \pm 0,51 \mathrm{~L} E E F^{(3)}$ & $-0,41$ & - \\
\hline $\mathrm{T} 20$ & - & - & - & $2,00 \pm 0,63 \mathrm{JL} E E F$ & 0,00 & - \\
\hline
\end{tabular}

${ }^{(1)}$ Médias ( \pm erro-padrão) seguidas de mesma letra não diferem entre si, a 5\% de probabilidade $(\mathrm{CV}=13,66 \%)($ letras maiúsculas comparam todos os tratamentos; letras minúsculas comparam cada agente de controle entre as três concentrações; e letras maiúsculas em itálico comparam os tratamentos na concentração menor, média e maior). ${ }^{(2)}$ TD, terra diatomácea; Bb, Beauveria bassiana; EM-4 ativado; quantidade (\%) dos agentes de controle, em relação a massa total do substrato $(60 \mathrm{~g})$, utilizada nos tratamentos. ${ }^{(3)}$ As letras EEF em T19 e T20 são as comparações para as concentrações menor, média e maior, respectivamente, entre todos os agentes de controle. 
significativo de eficiência de controle, quando os dois agentes são associados. Este aspecto pode ser explorado em programas de manejo de populações de cascudinho, incorporando-se os dois agentes de controle associados. Nas associações de TD + EM-4 (T10, T11 e T12), Bb + EM-4 (T13, T14 e T15) e $\mathrm{TD}+\mathrm{Bb}+\mathrm{EM}-4(\mathrm{~T} 16, \mathrm{~T} 17$ e T18), nas três concentrações, o efeito observado foi aditivo, o que confirma que a ação do EM-4 diminui a eficiência dos demais agentes de controle. Efeitos sinérgicos, decorrentes da associação de terra diatomácea e B. bassiana, também foram observados no controle de $R$. dominica (Lord, 2001), T. castaneum (Akbar et al., 2004) e A. diaperinus (Oliveira \& Alves, 2007).

Para os testes in vitro realizados com EM-4, não submetidos ao processo de filtração, não foi observada diferença significativa quanto ao número de conídios germinados no tratamento com EM-4 e na testemunha, com 97,94 e 98,22\% de germinação, respectivamente. Nos testes de UFC, crescimento vegetativo e produtividade de conídios, não ocorreu desenvolvimento do fungo, tendo-se observado no meio de cultura apenas colônias de bactérias e leveduras, o que pode ser explicado pela competição entre os microrganismos em estudo, já que o EM-4 é composto por bactérias ácido-láticas (Lactobacillus e Pediococcus), leveduras (Sacharomyces) e, em menor número, por bactérias fotossintéticas e actinomicetos, todos compatíveis entre si (Higa \& Parr, 1994). Antes de ser ativado e diluído, o produto comercial EM-4 apresenta uma concentração de leveduras com $2 \times 10^{6} \mathrm{UFC} \mathrm{mL}^{-1}$, na suspensão, e $1 \times 10^{5} \mathrm{UFC} \mathrm{mL}^{-1}$ de bactérias produtoras de ácidolático (Guim et al., 2002). O acelerado crescimento desses microrganismos pode ter influenciado, de

Tabela 2. Efeito do composto EM-4 ativado, submetido à filtração, sobre o fungo Beauveria bassiana, em quatro variáveis do desenvolvimento in vitro ${ }^{(1)}$.

\begin{tabular}{lrrr}
\hline Variável & \multicolumn{2}{c}{ Tratamentos } & CV (\%) \\
\cline { 2 - 3 } & \multicolumn{1}{c}{ Testemunha } & \multicolumn{1}{c}{ EM-4 } & \\
\hline Germinação (\%) & $97,39 \pm 1,00 \mathrm{a}$ & $96,97 \pm 1,09 \mathrm{a}$ & 2,24 \\
UFC & $114,40 \pm 2,03 \mathrm{a}$ & $116,20 \pm 2,51 \mathrm{a}$ & 4,44 \\
CV $\left(\mathrm{cm}^{2}\right)$ & $13,17 \pm 0,62 \mathrm{a}$ & $7,64 \pm 0,62 \mathrm{~b}$ & 9,60 \\
PD $\left(\mathrm{conídios} \mathrm{cm}^{-2}\right)$ & $39,01 \times 10^{7} \pm 4,49 \mathrm{a}$ & $18,77 \times 10^{7} \pm 2,99 \mathrm{~b}$ & 28,95 \\
\hline
\end{tabular}

${ }^{(1)}$ Médias ( \pm erro-padrão) seguidas de letra igual, na linha, não diferem entre si, a 5\% de probabilidade. ${ }^{(2)}$ UFC, unidades formadoras de colônias; $\mathrm{CV}$, crescimento vegetativo; $\mathrm{PD}$, produtividade de conídios. maneira negativa, o desenvolvimento do fungo, em consequiência da competição pelo substrato (Pelczar et al., 1980).

No teste de germinação em que se utilizou EM-4, submetido ao processo de filtração, não houve diferença estatística entre o tratamento com este produto e a testemunha, com viabilidade dos conídios superior a $96 \%$ (Tabela 2). Também não se observou diferença significativa quanto ao teste de UFC. As demais variáveis analisadas foram afetadas pela aplicação de EM-4, com redução de aproximadamente $42 \%$, em relação ao crescimento vegetativo, e de $52 \%$ na produtividade de conídios. Neste caso, desconsidera-se a existência de competição entre os microrganismos, em razão do processo de filtração, que eliminou as células reprodutoras. A ação inibidora de EM-4 sobre o desenvolvimento de $B$. bassiana pode ser atribuída aos metabólitos produzidos pelos microrganismos existentes no produto, ou ao valor de seu $\mathrm{pH}$, de aproximadamente 3,2 (Higa \& Parr, 1994), pois, segundo Hallsworth \& Magan (1996), a melhor faixa de $\mathrm{pH}$ para o desenvolvimento de $B$. bassiana situa-se entre 5 e 8.

Os resultados observados nesse teste auxiliam no entendimento dos resultados de mortalidade de A. diaperinus, em que se observou redução no controle da praga, nos tratamentos em que o EM-4 foi associado à $B$. bassiana, o que ressalta o efeito negativo de EM-4 sobre o fungo. Para os tratamentos em que o EM-4 foi associado à terra diatomácea, também houve redução na mortalidade, porém nesse caso, a água utilizada durante as pulverizações diárias de EM-4 pode ter contribuído com o aumento da umidade no recipiente e interferido no efeito da terra diatomácea, em razão da diminuição da abrasão e, em conseqüência, pode ter provocado menor efeito dessecante (Fields \& Korunic, 2000; Mewis \& Ulrichs, 2001). As partículas de terra diatomácea, ao absorver água, tornam-se menos eficazes (Arthur, 2001), assim, a terra diatomácea é mais eficiente em condições de clima seco (Atui et al., 2003).

Oliveira \& Alves (2007) verificaram que, para o controle de A. diaperinus, a eficiência da terra diatomácea, do fungo B. bassiana e da associação de ambos, foi maior em condições de baixa umidade relativa. Resultados semelhantes foram observados por Lord (2005), no controle de $R$. dominica com esses mesmos agentes. A variação de $10 \%$ na umidade relativa é suficiente para influenciar os resultados de mortalidade provocados por esses agentes, para o controle de T. confusum (Vayas \& Athanassiou, 2004). 


\section{Conclusões}

1. A utilização associada de terra diatomácea e Beauveria bassiana tem efeito não-aditivo sinérgico para o controle de Alphitobius diaperinus.

2. O composto EM-4 reduz a mortalidade de A. diaperinus se associado à terra diatomácea $\mathrm{e}$ B. bassiana.

3. Em testes in vitro, o composto EM-4 reduz o crescimento vegetativo e a produtividade de conídios de B. bassiana.

\section{Agradecimentos}

À Professora Inês Fonseca, do Departamento de Agronomia, da Universidade Estadual de Londrina, pelo auxílio nas análises estatísticas; ao Engenheiro Agrônomo Gilberto Yudi Shingo, e a empresa Mokiti Okada, por fornecer o produto EM-4.

\section{Referências}

AKBAR, W.; LORD, J.C.; NECHOLS, J.R.; HOWARD, R.W. Diatomaceous earth increases the efficacy of Beauveria bassiana against Tribolium castaneum larvae and increases conidia attachment. Journal of Economic Entomology, v.97, p.273-280, 2004.

ALVES, L.F.A.; BUZARELLO, G.D.; OLIVEIRA, D.G.P.; ALVES, S.B. Ação da terra de diatomácea contra adultos do cascudinho Alphitobius diaperinus (Panzer, 1797) (Coleoptera: Tenebrionidae). Arquivos do Instituto Biológico, v.73, p.115-118, 2006.

ALVES, L.F.A.; GASSEN, M.H.; PINTO, F.G.S.; NEVES, P.M.O.J.; ALVES, S.B. Ocorrência natural de Beauveria bassiana (Bals.) Vuilleman (Moniliales: Moniliaceae) sobre o cascudinho, Alphitobius diaperinus (Panzer) (Coleoptera: Tenebrionidae), em aviário comercial de Cascavel, PR. Neotropical Entomology, v.34, p.507-510, 2005.

ALVES, S.B. Fungos entomopatogênicos. In: ALVES, S.B. (Ed.). Controle microbiano de insetos. Piracicaba: Fealq, 1998. p.289381.

ANDRADE, M.S.; GÓES, M.A.C.; OLIVEIRA, N.M.M.; FRANÇA, S.C.A. Especiação de Sílica e Estudo de Metodologia de Digestão de Diatomito Assistida por Microondas com Radiação Focalizada. In: Congresso Anual da ABM Internacional, 56., 2001, Belo Horizonte. Anais. [Belo Horizonte: ABM Internacional, 2001.] p.1019-1028.

ARTHUR, F.H. Immediate and delayed mortality of Oryzaephilus surinamensis (L.) exposed on wheat treated with diatomaceous earth: effects of temperature, relative humidity, and exposure interval. Journal of Stored Products Research, v.37, p.13-21, 2001.

ATUI, M.B.; LAZZARI, F.A.; LAZZARI, S.M.N. Evaluation of the methodology for detection of diatomaceous earth residue in wheat grain and flour. Revista do Instituto Adolfo Lutz, v.62, p.11-16, 2003.

AXTELL, R.C.; ARENDS, J.J. Ecology and management of arthropod pest of poultry. Scientia Agricola, v.35, p.101-126, 1990.

FIELDS, P.; KORUNIC. Z. The effect of grain moisture content and temperature on the efficacy of diatomaceous earths from different geographical locations against stored-product beetles. Journal of Stored Products Research, v.36, p.1-13, 2000.

GUIM, A.; ANDRADE, P.; ITURRINO-SCHOCKEN, R.P.; FRANCO, G.L.; RUGGIERI, A.C.; MALHEIROS, E.B. Estabilidade aeróbica de silagens de capim-elefante (Pennisetum purpureum, Schum.) emurchecido e tratado com inoculante microbiano. Revista Brasileira de Zootecnia, v.31, p.21762185, 2002.

HALLSWORTH, J.E.; MAGAN, N. Culture age, temperature and $\mathrm{pH}$ affect the polyol and trehalose contents of fungal propagules. Applied and Environmental Microbiology, v.62, p.2435-2442, 1996.

HIGA, T. O meio ambiente do ponto de vista do mundo microscópico. In: Forum Global das Organizações Não-Governamentais. Rio de Janeiro: Fundação Mokiti Okada, 1992. 11p.

HIGA, T.; PARR, J.F. Beneficial and effective microorganisms for a sustainable agriculture and environment. Atami: International Nature Farming Research Center, 1994. 16p.

KOPPENHOFER, A.M.; BROWN, I.M.; GAUGLER, R.; GREWAL, P.S.; KAYA, H.K.; KLEIN, M.G. Synergism of entomopathogenic nematodes and imidacloprid against white grubs: greenhouse and field evaluation. Biological Control, v.19, p.245$251,2000$.

KORUNIC, Z. Review: diatomaceous earths, a group of natural insecticides. Journal of Stored Products Research, v.34, p.8797, 1998.

LORD, J.C. Desiccant dusts synergize the effect of Beauveria bassiana (Hyphomycetes: Moniliales) on stored-grain beetles. Journal of Economic Entomology, v.94, p.367-372, 2001.

LORD, J.C. Low humidity, moderate temperature, and desiccant dust favor efficacy of Beauveria bassiana (Hyphomycetes: Moniliales) for the lesser grain borer, Rhyzopertha dominica (Coleoptera: Bruchidae). Biological Control, v.34, p.180-186, 2005.

LORINI, I. Manual técnico para o manejo integrado de pragas de grãos de cereais armazenados. Passo Fundo: Embrapa Trigo, 2001. 80p.

MEWIS, I.; ULRICHS, C. Action of amorphous diatomaceous earth against different stages of the stored product pests Tribolium confusum, Tenebrio molitor, Sitophilus granarius and Plodia interpunctella. Journal of Stored Products Research, v.37, p.153164, 2001.

NAKANO, O.; SILVEIRA NETO, S.; ZUCCHI, R.A. Entomologia econômica. São Paulo: Livroceres, 1981. 314p.

O'CONNOR, J.P. Alphitobius diaperinus (Panzer) (Col.: Tenebrionidae) damaging polystyrene insulation in Irish piggery. Entomologist's Monthly Magazine, v.123, p.14721475, 1987. 
OLIVEIRA, D.G.P.; ALVES, L.F.A. Interação do fungo Beauveria bassiana (Bals.) Vuill. com terra diatomácea para o controle de Alphitobius diaperinus (Panzer) (Coleoptera: Tenebrionidae), o cascudinho-dos-aviários. BioAssay, v.2, p.1-6, 2007.

PELCZAR, M.; REID, R.; CHAN, E.C.S. Microbiologia. São Paulo: McGraw-Hill, 1980. 566p.

ROHDE, C.; ALVES, L.F.A.; BRESSAN, D.F.; NEVES, P.M.O.J.; SILVA, E.R.L.; ALVES, S.B.; ALMEIDA, J.E.M. Seleção de isolados de Beauveria bassiana (Bals.) Vuill. e Metarhizium anisopliae (Metsch.) Sorok. contra o cascudinho Alphitobius diaperinus (Panzer) (Coleoptera: Tenebrionidae). Neotropical Entomology, v.35, p.231-240, 2006.

VAYAS, B.J.; ATHANASSIOU, C.G. Factors affecting the insecticidal efficacy of the diatomaceous earth formulation SilicoSec against adults and larvae of the confused flour beetle, Tribolium confusum DuVal (Coleoptera: Tenebrionidae). Crop Protection, v.23, p.565-573, 2004.

Recebido em 31 de agosto de 2007 e aprovado em 19 de dezembro de 2007 